\title{
Intervencionismo coronario percutáneo en pacientes diabéticos: desafíos actuales y perspectivas futuras
}

\author{
Percutaneous coronary intervention in diabetic patients
}

\author{
Pablo Salinas, Carlos H. Salazar
}

\section{RESUMEN}

El intervencionismo coronario percutáneo en pacientes diabéticos se asocia a enfermedad multivaso, escenarios anatómicos más complejos y mayores retos técnicos. Aunque gracias a la evolución de las técnicas y materiales han mejorado los resultados, siempre han sido inferiores a los pacientes no diabéticos. En el terreno de la investigación, la evidencia todavía se basa en gran medida en análisis de subgrupos, contando con escasos estudios específicos de diabéticos. En esta revisión se analizan cuáles son los desafíos a los que se afronta el cardiólogo intervencionista, los recursos disponibles para enfrentarlos de acuerdo con la evidencia actual, y las perspectivas futuras sobre esta materia.

Palabras claves: diabetes, stents, cirugía coronaria, enfermedad coronaria.

\begin{abstract}
Percutaneous coronary intervention in diabetic patients is associated with multivessel disease, highly complex coronary anatomy and greater technical challenges. Although thanks to the evolution of techniques and devices the results have been improved, they have always been inferior to non-diabetic patients. Data from research is still largely based on subgroup analysis, with few specific studies of diabetics. In this review we analyze which are the current challenges that the interventional cardiologist have to confront, the resources available to face them according to current evidence and the future perspectives on this matter
\end{abstract}

Keywords: diabetes, stetnt, $C A B G$, coronary disease.

Revista Argentina de Cardioangiología Intervencionista 2018;9(4):198-203. DOl: 10.30567/RACI/201804/0198-0203

\section{INTRODUCCIÓN}

El paciente diabético tiene una gran relevancia en los laboratorios de hemodinámica. Actualmente representan entre el 20-30\% de los pacientes que son referidos a intervención coronaria percutánea (ICP) a nivel mundial ${ }^{1}$. La proyección de la enfermedad diabética, especialmente la diabetes tipo 2, es de amplio crecimiento. En el último informe de la organización mundial de la salud (OMS) se calcula que 422 millones de adultos tenían diabetes en 2014, con un ascenso de la prevalencia llegando al $8,5 \%^{2}$.

Aunque las guías de práctica clínica tienden a recomendar la revascularización quirúrgica sobre el $\mathrm{ICP}^{3}$, en la práctica clínica existen numerosos factores de hacen que el ICP sea el tratamiento finalmente realizado. Estos factores son: la presentación clínica como infarto agudo de miocardio con elevación del ST, la enfermedad monovaso o multivaso sin compromiso de la descendente anterior, malos lechos distales, tratamiento de injertos fallidos, comorbilidades que condicionan un elevado riesgo quirúrgico, o incluso la preferencia del paciente. Es difícil ofrecer cifras fiables por la falta de estudios específicos, pero se estima que entre un 40-60\% de los pacientes con indicación de revascularización son finalmente tratados por ICP. En nuestro centro hemos analizado una serie consecutiva de primer diagnóstico de enfermedad coronaria en 5370 pacientes diabéticos no valvulares (2004-2018) y el tratamiento finalmente rea-

Hospital Clínico San Carlos. Madrid, España.

$\triangle$ Correspondencia: Pablo Salinas. Cardiología Intervencionista. Hospital Clínico San Carlos. Profesor Martín Lagos SN. 28040. Madrid, España.

Email: salinas.pablo@gmail.com. Tel+34913303283.Fax+34913303291 Conflicto de intereses de los autores: Pablo Salinas ha recibido honorarios como orador en conferencias educativas para Alvimedica, Biomenco, Terumo, Boston Scientific.

Recibido:08/11/2018|Aceptado: 29/11/2018 lizado fue la cirugía en un $13 \%$, ICP en un $63 \%$ y tratamiento médico en un $24 \%$ (datos no publicados).

El ICP en pacientes diabéticos presenta un escenario de gran complejidad por diversos factores (Figura 1). Las características anatómicas de la enfermedad coronaria del paciente diabético son de mayor dificultad: afectación difusa (multivaso, multisegmento), vasos finos con enfermedad difusa y escasos segmentos libres de enfermedad, mayor presencia de fibroateroma de cápsula fina y mayor calcificación. Estas complejidades anatómicas demandan altos requerimientos técnicos para el ICP (manejo de bifurcaciones, oclusiones crónicas, enfermedad difusa, técnicas de modificación de placa calcificada, etc.). La enfermedad tiene un desarrollo precoz con un aumento de la probabilidad futura de nuevas intervenciones, y con una progresión acelerada comparada con pacientes no diabéticos. La respuesta a las terapias intracoronarias como angioplastia con balón y stent presenta peores resultados tanto clínicos como angiográficos en el medio y largo plazo, incrementando las tasas del fallo del stent (trombosis y reestenosis), y por tanto las tasas de nueva revascularización. Los pacientes diabéticos se acompañan de otros factores de riesgo y comorbilidades (obesidad, dislipemia, enfermedad renal) que de forma sinérgica contribuyen a potenciar los factores previamente comentados. Este acúmulo de factores interrelacionados y que se favorecen entre sí conduce a peores resultados clínicos (evento combinado definido clásicamente como muerte, infarto, nueva revascularización), no ya solo del ICP sino también del tratamiento médico o quirúrgico de la enfermedad coronaria.

De todos estos desafíos a los que nos enfrentamos cuando debemos tratar la enfermedad coronaria en el paciente diabético, en esta revisión nos centraremos en aquellos con los que específicamente debe lidiar el cardiólogo intervencionista, proponiendo un enfoque práctico y ofreciendo soluciones acordes a la evidencia actual. 


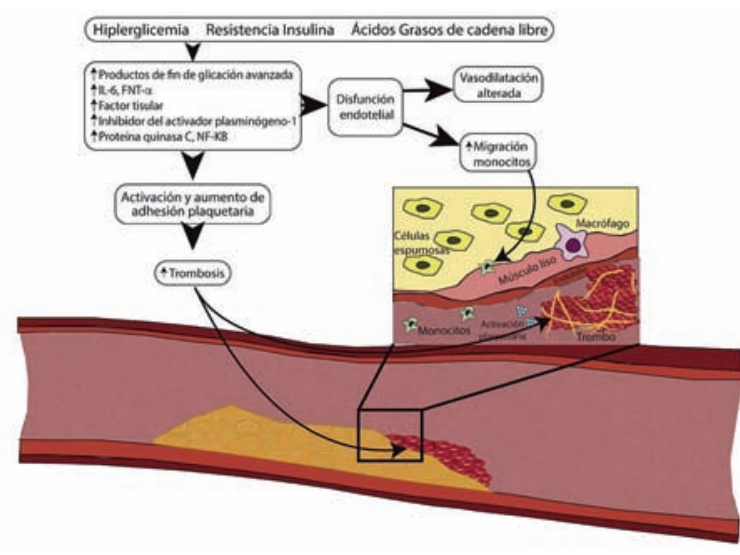

Figura 1. Mecanismos implicados en la aterosclerosis acelerada en el paciente diabético.

\section{COMPLEJIDAD ANATÓMICA}

La anatomía compleja de la enfermedad coronaria en los pacientes diabéticos es un hallazgo no modificable en el momento del diagnóstico y frecuentemente dirige el paciente hacia cirugía de revascularización coronaria (en las últimas guías de la AHA / ACC sólo los pacientes con enfermedad de uno o dos vasos en la que no participe la descendente anterior son dirigidos a intervencionismo).

Sin embargo, en el ICP no diabético tenemos evidencia firme que el uso sistemático de guía de presión puede reducir los eventos combinados en pacientes multivaso ${ }^{4,5}$. Si bien no disponemos de estudios específicos de ICP multivaso guiada por fisiología coronaria en pacientes diabéticos, la presencia de diabetes no parece modificar la capacidad diagnóstica de los índices de reserva de flujo. El primer razonamiento lógico se dirige hacia optimizar el número de vasos necesarios a tratar; las técnicas de fisiología intracoronaria (iFR / FFR) son capaces de reclasificar pacientes con enfermedad de tres vasos angiográfica en enfermedad monovaso o sin indicación quirúrgica, reduciendo considerablemente el número de vasos a tratar y el número de stents a implantar. Por ejemplo, en el estudio SYNTAX II realizado en pacientes con enfermedad de tres vasos elegibles para ICP, se evaluaban por protocolo todas las lesiones consideradas angiográficamente gra$\mathrm{ves}^{5}$. En este estudio, partiendo de 3,49 lesiones por paciente inicialmente seleccionadas para ICP por angiografía, solo un $74,6 \%$ (2,64 lesiones por paciente) fueron funcionalmente significativas, motivando que solo un $37,2 \%$ de los pacientes precisaran ICP en los tres vasos. El número de stents por paciente, comparado con el estudio SYNTAX I fue también significativamente menor (3,78 en SYNTAX II frente a 5,19 en SYNTAX I, $\mathrm{p}<0,001)$. Los resultados del evento combinado fueron mejores que la cohorte histórica de intervencionismo en SYNTAX I y comparables a la cohorte histórica de cirugía coronaria en SYNTAX I. Entendiendo la limitación que no es un estudio específicamente realizado en diabéticos (el estudio contaba con un 30,3\% de diabéticos y no halló heterogeneidad de resultados según el paciente fuese diabético o no), la aplicación de estas estrategias de fisiología podría reducir el número de vasos y lesiones a tratar con seguridad y de esta manera simplificar el ICP. En opinión de los autores, el intervencionismo guiado por fisiología coronaria debería tener un papel crucial en la selección de las lesiones a tratar, sobre todo en pacientes multivaso, e independientemente de la severidad angiográfica de la lesión ${ }^{6}$. El avance de la fisiología en los laboratorios de cardiología intervencionista aún no tiene el impacto suficiente entre la comunidad, pero está en aumento gracias a las mejoras tecnológicas que facilitan la evaluación sin adenosina como lo es el iFR ${ }^{7}$, e incluso sin instrumentación intracoronaria $(\mathrm{QFR})^{8}$. Estas técnicas pueden ayudar también en la evaluación de enfermedad multisegmento y difusa, logrando reducir el número y longitud de los stents a implantar?

Una vez seleccionadas y delimitadas las lesiones a tratar se debería optimizar adecuadamente la implantación de los stents. Para ello es fundamental la preparación de la placa, elegir buenas zonas de aterrizaje de los stents y valorar correctamente los diámetros de referencia. Cuanto mejor sea la preparación de placa menor será la probabilidad de infraexpansión; se sabe que mayores diámetros intraluminales se asocian con menor tasa del fallo del stent. Eligiendo adecuadamente las "landing zones" y evaluando adecuadamente los diámetros evitaremos la elección inadecuada del tamaño del stent y minimizaremos las zonas de malaposición. Dado que la angiografía puede ser limitada para estimar correctamente los diámetros de referencia en presencia de enfermedad difusa, la imagen intracoronaria cobra especial relevancia. El uso de ecografía intravascular (IVUS) guiando el implante de stents liberadores de fármaco (DES) puede reducir la tasa combinada de eventos cardiovasculares, especialmente el componente de nueva revascularización ${ }^{10}$. La tomografía de coherencia óptica se ha mostrado no inferior al IVUS en la optimización del implante de $\mathrm{DES}^{11}$.

\section{PROGRESIÓN ACELERADA DE LA ENFERMEDAD CORONARIA}

En la población general, los casos de angina recurrente son más frecuentemente causados por la progresión de enfermedad aterosclerótica que por fallo de la revascularización previa $^{12}$. Tras un procedimiento de revascularización, ¿cómo podemos frenar la progresión de aterosclerosis? La primera medida serían las líneas generales de prevención secundaria según las guías actuales de práctica clínica: antiagregación, estatinas de alta potencia, betabloqueantes e inhibidores de la enzima convertidora de angiotensina (o inhibidores del receptor de angiotensina), entre otros, en sus indicaciones apropiadas ${ }^{13}$.

Requiere una atención especial el tratamiento hipolipemiante, uno de los factores de riesgo modificables donde la intervención farmacológica ha logrado mejores resultados en reducción de eventos cardiovasculares, con evidencia procedente de más de 75.000 pacientes evaluados en múltiples estudios ${ }^{14}$. La presencia de enfermedad cardiovascular establecida en pacientes con diabetes obliga a buscar un objetivo de colesterol-LDL $<70 \mathrm{mg} / \mathrm{dl}$ inicialmente con altas dosis de estatinas $^{14,15}$. En los pacientes que no se logra el objetivo, la combinación con otros agentes como ezetimibe, inhibidores de PCSK9 (especialmente evolocumab) o anacetrapib han demostrado reducir los eventos cardiovasculares, confirmándose los hallazgos en subanálisis de pacientes diabéticos ${ }^{16-18}$ Específicamente relacionado con la progresión de enfermedad aterosclerótica, el inhibidor de PCSK9 evolocumab asociado a estatinas logró una mayor regresión de placa que el grupo control solo con estatinas ${ }^{19}$. 
Como segunda medida se debe realizar un adecuado control glicémico. De forma genérica para pacientes no embarazadas se recomienda un objetivo de hemoglobina glicada $<7 \%$. Los pacientes con menor edad y tiempo de evolución de diabetes, sin historia de hipoglucemias y sin ateroesclerosis avanzada o comorbilidades graves podrían beneficiarse de un control más estricto ${ }^{20}$. La demostración de reducción de eventos o muerte cardiovascular con tratamiento intensivo ha sido más compleja, pero si bien existe cierta heterogeneidad entre estudios ${ }^{21}$, se ha logrado demostrar reducción de eventos cardiovasculares en el seguimiento extendido de al menos dos estudios ${ }^{22,23}$

Más allá del objetivo de hemoglobina glicada (que debe ser individualizado), existen antidiabéticos específicos que han demostrado reducción de eventos cardiovasculares. En pacientes con diabetes tipo 2 y enfermedad cardiovascular establecida, acompañando a la modificación de estilo de vida ${ }^{24}$ y a la metformina, se recomienda uno de los tres fármacos que han demostrado reducir los eventos cardiovasculares: empaglifozina, liraglutide, canaglifozina. Especialmente interesantes parecen la empaglifozina y liraglutide, que redujeron la mortalidad cardiovascular y el liraglutide además el infarto de miocardio ${ }^{25}$.

Otro fármaco atractivo por su novedosa diana terapéutica es el canakinumab, un inhibidor de la interleukina $1 b$ que ha demostrado reducir eventos cardiovasculares en pacientes con infarto previo y elevación de proteína $\mathrm{C}$ reactiva. Si bien no logró demostrar la hipótesis de reducción de nuevo diagnóstico de diabetes, los resultados fueron comparables en el subgrupo de diabéticos ${ }^{26}$.

\section{FALLO DEL STENT}

El término fallo del stent ("stent failure") comprende la trombosis, la reestenosis y la oclusión crónica intrastent (en este último caso el mecanismo puede ser indeterminado). En los pacientes diabéticos cualquiera de estas formas es mucho más frecuente que en pacientes no diabéticos, principalmente motivado por factores protrombóticos y proproliferativos que se resumen en la Figura $\mathbf{2} \mathbf{A}^{27}$. Los factores mecánicos están presentes en un grandísimo porcentaje de casos de fallo del stent, y se ven indirectamente favorecidos por la diabetes al tener lesiones más complejas y por tanto obteniendo con mayor frecuencia resultados subóptimos (Figura 2B).

Si bien el fallo del stent es un concepto fácil de entender, su cuantificación en los estudios es más compleja. La trombosis se ha definido según la Academic Research Consortium con unos criterios ampliamente aceptados. En cuanto a la reestenosis, los estudios clásicos que realizaban seguimiento angiográfico definieron reestenosis como una estenosis $\geq 50 \%$ intrastent o en los $5 \mathrm{~mm}$ del borde, independientemente de que causara o no síntomas. Sin embargo, este hallazgo generaba en un alto porcentaje de casos una nueva revascularización no necesariamente justificada ${ }^{28}$. En los estudios más modernos se suele utilizar como evento la "nueva revascularización de lesión diana clínicamente indicada” o bien el más amplio evento combinado "fallo de lesión diana" que usualmente comprende nueva revascularización de lesión diana, infarto de miocardio (no atribuible a vaso no-diana) o muerte cardiaca. Estos eventos, más pragmáticos y con mayor traducción clínica, sin embargo, no cuantifican los fallos del stent silentes (casos asintomáticos que se pueden detectar en un cateterismo posterior) o aquellos en los que no
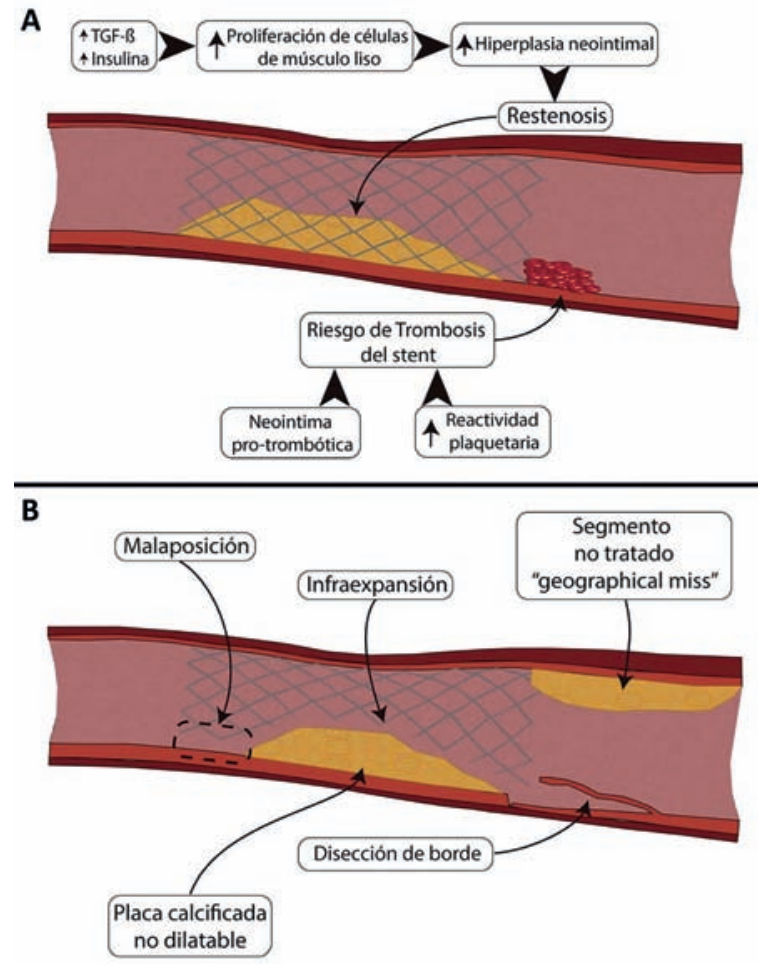

Figura 2. Mecanismos asociados a fallo del stent. A: mecanismos acrecentados en pacientes diabéticos. $B$ : factores mecánicos.

se decide una nueva revascularización (por ejemplo, por su elevada complejidad como puede ser una reestenosis oclusiva completa.

En cuanto a la trombosis del stent, las estrategias actuales para evitarla se podrían resumir en tres. La primera sería elegir una plataforma de última generación, con polímero biocompatible, diseño de celda abierta y strut fino ${ }^{29,30}$. La segunda sería usar, dentro de sus indicaciones, nuevos inhibidores P2Y12 como ticagrelor o especialmente prasugrel (mayor eficacia en diabéticos, con NNT más bajo que en no diabéticos) en lugar de clopidogrel $^{31,32}$. Finalmente, y especialmente en lesiones complejas, el uso de imagen intracoronaria para guiar la ICP podría reducir los factores mecánicos asociados con la trombosis (malaposición, distorsión de la arquitectura del stent, etc. $)^{33,34}$.

En cuanto a la eficacia medida en términos de reestenosis o nueva revascularización de vaso diana, hemos visto cómo las nuevas tecnologías de ICP han mejorado los resultados en el contexto del paciente diabético. El salto desde la angioplastia con balón al stent convencional redujo las tasas de restenosis de casi un $60 \%$ a un $25 \%$, y la aparición de la primera generación de DES la redujo a su vez hasta cerca de un $10 \%$. La segunda generación de DES, si bien ha reducido las tasas de trombosis ${ }^{30}$ comparado con la primera, arroja resultados no claramente superiores en términos de eficacia. En la Tabla 1 se muestran los resultados más relevantes de estudios con DES de segunda generación en diabéticos. Una de las razones de esta aparente meseta en la reducción de la restenosis que han encontrado los DES puede estar en la resistencia relativa de la célula endotelial diabética a la rapamicina. Actualmente los análogos de la rapamicina (-limus) son los fármacos utilizados en la práctica totalidad de los stents comercializados actualmente debido a su eficacia inhibiendo la proliferación endotelial. Sin embargo, existen datos que demuestran que la célula endotelial diabética tiene, comparada con la célula no diabética, una resistencia relativa a los análo- 
TABLA 1. Principales estudios realizados con stents en población diabética

\begin{tabular}{|c|c|c|c|c|c|c|c|c|c|c|}
\hline ESTUDIO & STENTS & $\mathrm{N}$ & SEG & TIPO & MACE† & MUERTE & IAM & TLR & TVR & TROMBOSIS $\neq$ \\
\hline \multicolumn{11}{|l|}{ Short term } \\
\hline ESSENCEDIABETES ${ }^{38}$ & EES vs SES & 300 & 1 año & ECR & $2.0 \%$ vs $5.3 \%$ & $1.3 \%$ vs $3.3 \%$ & $0 \%$ vs $1.3 \%$ & $0.7 \%$ vs $2.6 \%$ & $0.7 \%$ vs $4.0 \%$ & $0.7 \%$ vs $0.7 \%$ \\
\hline EXCELLENT ${ }^{39}$ & EES vs ZES & 1855 & 1 año & Cohorte & - & $3.0 \%$ vs $2.8 \%$ & $0.8 \%$ vs $0.6 \%$ & $1.7 \%$ vs $1.8 \%$ & $2.4 \%$ vs $3.5 \%$ & $0.8 \%$ vs $0.1 \%$ \\
\hline SORT OUT IV ${ }^{40}$ & EES vs SES & 390 & $18 \mathrm{~m}$ & Post-hoc ECR & - & $3.6 \%$ vs $8.2 \%$ & $0.5 \%$ vs $3.6 \%$ & $3.1 \%$ vs $7.7 \%$ & $6.7 \%$ vs $10.7 \%$ & $1.0 \%$ vs $2.6 \%$ \\
\hline SPIRITV Vi1 & EES vs PES & 324 & 1 año & ECR & - & $1.4 \%$ vs $2.9 \%$ & $3.3 \%$ vs $8,7 \%$ & $11.2 \%$ vs $6.7 \%$ & $9.8 \%$ vs $5.8 \%$ & $0 \%$ vs $1.9 \%$ \\
\hline TUXEDO ${ }^{42}$ & PES vs EES & 1830 & 1 año & ECR & $\begin{array}{l}\text { DMID: } 8.2 \text { vs } 3.9 \% \\
\text { NDMID: } 4.4 \text { vs } 3.0 \%\end{array}$ & $\begin{array}{l}\text { DMID: } 3.5 \text { vs } 3.1 \% \\
\text { NDMID: } 1.6 \text { vs } 1.7 \% \\
\end{array}$ & $\begin{array}{l}\text { DMID: } 4.4 \text { vs } 1.3 \% \\
\text { NDMID: } 2.4 \text { vs } 1.1 \%\end{array}$ & $\begin{array}{l}\text { DMID: } 5.2 \text { vs } 1.0 \% \\
\text { NDMID: } 2.2 \text { vs } 1.3 \% \\
\end{array}$ & $\begin{array}{l}\text { DMID: } 4.1 \text { vs } 0.5 \% \\
\text { NDMID: } 2.0 \text { vs } 0.6 \%\end{array}$ & $\begin{array}{l}\text { DMID: } 3.0 \% \text { vs } 0.5 \% \\
\text { NDMID: } 1.5 \text { vs } 0.4 \%\end{array}$ \\
\hline \multicolumn{11}{|l|}{ Long term } \\
\hline RESOLUTE program ${ }^{43}$ & ZES & 878 & 2 años & Cohorte & $11,30 \%$ & $4,90 \%$ & $2.30 \%$ & $4.8 \%$ & $7.9 \%$ & $0.3 \%$ \\
\hline RESET and NEXT ${ }^{44}$ & EES/BES vs. SES & 2924 & 3 años & ECR & - & - & - & \begin{tabular}{|l|}
$10.1 \%$ (SES) vs $8.8 \%$ \\
(EES) vs $8.3 \%(\mathrm{BES})$ \\
\end{tabular} & - & \begin{tabular}{|l|}
$0.7 \%$ (SES) vs $0.7 \%$ \\
(EES) vs $0.4 \%(\mathrm{BES})$ \\
\end{tabular} \\
\hline ISARTEST $4^{45}$ & SES vs EES & 377 & 3 años & Post-hoc ECR & - & $16 \%$ vs $10 \%$ & $5.2 \%$ vs $4.3 \%$ & $16.6 \%$ vs $14.7 \%$ & & $4.1 \%$ vs $1.6 \%$ \\
\hline ISAR-TEST $5^{46}$ & PF-SES vs. ZES & 870 & 5 años & Post-hoc ECR & - & $24.4 \%$ vs $27.8 \%$ & $6.5 \%$ vs $7.6 \%$ & $18.6 \%$ vs $18.8 \%$ & $26.8 \%$ vs $26.2 \%$ & $2.5 \%$ vs $2.6 \%$ \\
\hline SORT OUT III ${ }^{47}$ & ZES vs SES & 337 & 5 años & Post-hoc ECR & $28.4 \%$ vs $18.5 \%$ & $16 \%$ vs $17.9 \%$ & $8.9 \%$ vs $7.1 \%$ & $14.8 \%$ vs $4.8 \%$ & $18.9 \%$ vs $8.3 \%$ & $1 \%$ vs $2.3 \%$ \\
\hline DiabeDES IV48 & EES vs SES & 213 & 4 años & ECR & $20.4 \%$ vs $23.8 \%$ & $12 \%$ vs $9.5 \%$ & $1.9 \%$ v $7.6 \%$ & $5.6 \%$ vs $9.5 \%$ & $12 \%$ vs $16.2 \%$ & $0.9 \%$ vs $1.9 \%$ \\
\hline ENDEAVOR III and IV49 & ZES vs SES/PES & 536 & 5 años & Post-hoc ECR & $17.7 \%$ vs $26.6 \%$ & $7.6 \%$ vs $15 \%$ & $1.3 \%$ vs $5.1 \%$ & 11.2 vs $12 \%$ & $17.2 \%$ vs $20 \%$ & $1.2 \%$ vs $0.8 \%$ \\
\hline SPIRIT II-IV ${ }^{50}$ & EES vs PES & 4989 & 3 años & Meta-análisis & $9,4 \%$ vs $13 \%$ & $3.2 \%$ vs $5.1 \%$ & $3.2 \%$ vs $5.1 \%$ & $6.0 \%$ vs $8.2 \%$ & $9.9 \%$ vs $11.4 \%$ & $0.7 \%$ vs $1.7 \%$ \\
\hline
\end{tabular}

† La definición de MACE varía entre estudios ¥ Trombosis definitiva o probable según la Academic Research Consortium.

SEG, seguimiento. m: meses. MACE: major adverse cardiac event. IAM: infarto agudo de miocardio. TLR: target lesion revascularization; TVR, target vessel revascularization; ECR, ensayo clínico randomizado; EES, everolimus eluting stent; SES, sirolimus-eluting stent; PES, paclitaxel-eluting stent; BES, biolimus-eluting stents; PF, polymer-free; ZES, zotarolimus-eluting stents;

gos de rapamicina que parece mediada por una disregulación de la proteína mammalian target of rapamycin $(\mathrm{mTOR})^{35}$. Se estima que se necesitarían casi 10 veces más dosis de rapamicina para obtener una respuesta similar a la célula no diabética. La leptina, una hormona liberada en mayor cantidad en pacientes con sobrepeso u obesidad (comorbilidad presente en un $85 \%$ de diabéticos ${ }^{36}$ ), tiene efectos directos facilitando la proliferación celular, limitando el efecto antiproliferativo de los análogos de la rapamicina ${ }^{37}$.

¿Cómo podremos mejorar las tasas de restenosis? Una de las estrategias más lógicas sería intentar afrontar la "resistencia al -limus" de la célula diabética intentando aumentar la dosis de droga que recibe la célula endotelial. Esta estrategia tiene la dificultad de que variar la proporción polímero/droga tiene los riesgos potenciales de aumentar el grosor del polímero y por tanto del strut y/o afectar a la cinética de liberación de la droga, lo que podría modificar negativamente la eficacia y seguridad del stent (dos soluciones innovadoras en estudio se describen más abajo en el artículo). Adicionalmente se debe recomendar la optimización tanto del número de stents (por ejemplo, con fisiología coronaria) como del propio implante del stent (por ejemplo, con imagen intracoronaria en casos complejos) para contribuir a reducir la reestenosis. Finalmente, se debe insistir en que es necesario un esfuerzo de investigación dirigido específicamente a pacientes diabéticos si queremos continuar mejorando los resultados de la ICP en diabéticos para continuar compitiendo con la cirugía de revascularización.

\section{PERSPECTIVAS FUTURAS}

Varias terapias farmacológicas se están evaluando en pacientes diabéticos para mejorar la evolución de la cardiopatía isquémica y los resultados de la revascularización percutánea. Nuevas dianas celulares relacionadas con la aterogénesis ${ }^{51}$ y con la proliferación de células endoteliales ${ }^{52,53}$, así como nuevas técnicas de modificación de placa ${ }^{54}$ podrán mejorar los resultados también en pacientes diabéticos.

En relación con los estudios de intervencionismo (Tabla 2) resulta interesante, aunque existen pocos datos aún, el estudio de estrategia híbrida en el que la se implanta una mamaria en la descendente anterior y se revasculariza el resto del árbol coronario con stents (estrategia de revascularización híbrida). En cuanto a los estudios con stents en diabéticos, se ensayan dos tecnologías dirigidas a incrementar la dosis de -limus en la célula endotelial. Una es con el Abluminus DES (Envision Scientific) en el que un stent convencional recibe un recubrimiento abluminal de sirolimus que también se aplica en las partes expuestas y en los extremos $(0.5 \mathrm{~mm})$ del balón de liberación. De esta manera se logra aplicar una dosis mayor de sirolimus en el implante.

Otra tecnología es el amphilimus, una formulación de sirolimus unida a ácidos grasos. Esta formulación tiene un diseño "inteligente", ya que al estar incrementada la actividad de los receptores de ácidos grasos en la membrana celular de las células diabéticas, se logra incrementar la dosis de sirolimus de forma diferencial a la célula no diabética que recibiría una dosis estándar ${ }^{55}$. Esta tecnología, aplicada en el stent Cre8Evo(Alvimedica), que es un strut sin polímero con arquitectura de celda abierta y strut fino, ha demostrado eficacia similar o superior a los actuales DES en un extenso programa de desarrollo clínico. En el estudio SUGAR actualmente en reclutamiento, se comparará con el stent Resolute Onyx (Medtronic) con objetivos clínicos de mejora de la eficacia medida como fallo de lesión diana. Próximo a iniciarse también está el estudio Diab8 en el que el mismo stent se enfrentará al stent Xience (Abbott Vascular), también con objetivos clínicos (revascularización de lesión diana) Los resultados de estos estudios constituirán uno de los mayores volúmenes de datos contemporáneos de ICP en paciente diabético con DES de segunda generación.

\section{CONCLUSIÓN}

El intervencionismo coronario percutáneo en pacientes diabéticos es complejo y un área donde se deben superar los resultados actuales. Tres áreas fundamentales son objeto de investigación: la complejidad anatómica, la aterosclerosis acelerada y el fallo del stent. El manejo del paciente multivaso (dónde y cómo tratar), los nuevos tratamientos médicos acompañantes al tratamiento revascularizador y la continua investigación en tecnología de stents aseguran un futuro prometedor del intervencionismo percutáneo en el paciente diabético. 
TABLA 2. Estudios en fase de diseño o reclutamiento en pacientes diabéticos con enfermedad coronaria.

\begin{tabular}{|c|c|c|c|c|c|c|}
\hline $\begin{array}{l}\text { ESTUDIO } \\
\text { CLINICO }\end{array}$ & DESCRIPCIÓN & NCT & $\begin{array}{l}\text { FECHA } \\
\text { FIN }\end{array}$ & $\begin{array}{l}\text { TIPODE } \\
\text { ESTUDIO }\end{array}$ & $\begin{array}{c}\text { TAMAÑO } \\
\text { MUESTRAL }\end{array}$ & OBJETIVO PRIMARIO \\
\hline \multicolumn{7}{|c|}{ Tratamiento médico } \\
\hline BETONMACE & RVX000222 (inhibidor proteínas BET) vs. placebo & 2586155 & 2018 & ECR & 2400 & MACE (120 semanas) \\
\hline Alopurinol & $\begin{array}{c}\text { Alopurinol en enfermedad multivaso revascularizada } \\
\text { mediante CABG o ICP vs. tratamiento estándar }\end{array}$ & 3700645 & 2020 & $E C R$ & 100 & Cambio en marcadores inflamatorios \\
\hline Sitagliptina & $\begin{array}{l}\text { Sitagliptina vs. acarbosa } \\
\text { en diabéticos con enfermedad coronaria }\end{array}$ & 3602638 & 2020 & $\mathrm{ECR}$ & 300 & $\begin{array}{l}\text { Cuantificación de calcificación } \\
\text { coronaria por tomografía computarizada }\end{array}$ \\
\hline ANDAMAN & $\begin{array}{l}\text { Aspirina } 100 \mathrm{mg} / 12 \mathrm{~h} \text { vs. } 100 \mathrm{mg} / 24 \mathrm{~h} \\
\text { post síndrome coronario agudo }\end{array}$ & 2520921 & 2020 & $E C R$ & 2574 & MACE vascular (18 meses) \\
\hline TRIMETA & Trimetazidina vs. placebo post-ICP & 3715582 & 2020 & ECR & 120 & Troponina y CPK pico \\
\hline \multicolumn{7}{|c|}{ Tratamiento revascularizador } \\
\hline HYCARDS & $\begin{array}{c}\text { Revascularización MICS en DA + ICP en otros vasos } \\
\text { vs. CABG convencional }\end{array}$ & 2504762 & 2019 & ECR & - & Eficacia \\
\hline COMBINE & $\begin{array}{l}\text { Estudio de evolución natural de lesiones interme- } \\
\text { dias con y sin TCFA, evaluadas con FFR y OCT }\end{array}$ & 2989740 & 2020 & $\mathrm{ECR}$ & 500 & MACE relacionado con lesión diana \\
\hline SUGAR & Estudio all comers ICP con PF-AES vs. PP-ZES & 3321032 & 2021 & ECR & 1164 & $\begin{array}{l}\text { TLF a } 1 \text { año (no inferioridad) } \\
\text { y TLF a } 2 \text { años (superioridad) }\end{array}$ \\
\hline $\begin{array}{l}\text { Abluminus } \\
\text { DES }\end{array}$ & ICP con Abluminus DES vs. EES & 3399994 & 2020 & $\mathrm{ECR}$ & 165 & $\begin{array}{l}\text { Volumen neointimal } \\
\text { intrastent ( } 9 \text { meses) }\end{array}$ \\
\hline DEDICATE & Registro poscomercialización Abluminus & 3509532 & 2022 & Registro & 1000 & TLF a 1 año \\
\hline
\end{tabular}

tConsulta de www.clinicaltrials.gov el 5-11-2018 con los criterios de coronary artery disease y diabetes mellitus, excluyendo aquellos con tamaño muestral <100. NCT, number clinical trials; BET, bromo- and extra-terminal domain; ECR, ensayo clínico randomizado; MACE, major adverse cardiac event; MICS, minimally invasive cardiac surgery; TCFA, thin cap fibroatheroma; FFR, fractional flow reserve; OCT, optical coherence tomography; TLF, target lesion failure: PF-AES, polymer-free amphilimus-eluting stents; PP-ZES, permanent polymer zotarolimus-eluting stents; EES, everolimus eluting stent.

\section{BIBLIOGRAFÍA}

1. FarkouhME, DomanskiM, Sleeper LA, etal. Strategiesfor multivessel revascularization in patients with diabetes. N Engl J Med. 2012;367(25):23752384. doi:10.1056/NEJMoa1211585

2. WHO | Global report on diabetes. WHO. http://www.who.int/diabetes/ global-report/en/. Accessed October 24, 2018.

3. Neumann F-J, Sousa-Uva M, Ah/sson A, et al. 2018 ESC/EACTS Guidelines on myocardial revascularization. Eur Heart J. doi:10.1093/eurheartj/ehy394

4. Tonino PAL, De Bruyne B, Pijls NHJ, et al. Fractional Flow Reserveversus Angiography for Guiding Percutaneous Coronary Intervention. N Engl J Med. 2009:360(3):213-224. doi:10.1056/NEJMoa0807611

5. Escaned J, Collet C, Ryan N, et al. Clinical outcomes of state-of-the-artpercutaneous coronary revascularization in patients with denovo threevessel disease: 1-yearresults of the SYNTAXIIstudy. EurHeartJ.2017;38(42):31243134. doi:10.1093/eurheartj/eh $\times 512$

6. Xaplanteris $P$, Fournier S, Pills NHJ, et al. Five-Year Outcomes with $P C 1$ Guided by Fractional Flow Reserve. N Engl J Med. 2018;379(3):250-259. doi:10.1056/NEJMoa1803538

7. Davies JE, Sen S, Dehbi H-M, et al. Use of the Instantaneous Wave-free Ratio or Fractional Flow Reserve in PCI. N Engl J Med. 2017;376(19):18241834. doi:10.1056/NEJMoa1700445

8. Xu B, Tu S, Qiao S, et al. Diagnostic Accuracy of Angiography-Based Quantitative Flow Ratio Measurements for Online Assessment of Coronary Stenosis. J Am Coll Cardiol. 2017;70(25):3077-3087. doi:10.1016/j.jacc.2017.10.035

9. Kikuta Y, Cook CM, Sharp ASP, et al. Pre-Angioplasty Instantaneous Wave-Free Ratio Pullback Predicts Hemodynamic Outcome In Humans With Coronary Artery Disease: Primary Results of the International MulticenteriFR GRADIENTRegistry. JACC Cardiovasc Interv. 2018;11(8):757-767. doi:10.1016/i.jcin.2018.03.005

10. Elgendy IY, Mahmoud AN, Elgendy AY, Bavry AA. Outcomes With Intravascular Ultrasound-Guided Stent Implantation: A Meta-Analysis of Randomized Trials in the Era of Drug-Eluting Stents. Circ Cardiovasc Interv. 2016;9(4):e003700. doi:10.1161/CIRCINTERVENTIONS.116.003700

11. Ali ZA, Maehara A, Généreux P, et al. Optical coherence tomography compared with intravascular ultrasound and with angiography to guide coronary stent implantation (ILUMIEN III: OPTIMIZE PCI): a randomised controlled trial. The Lancet. 2016;388(10060):2618-2628. doi:10.1016/501406736(16)31922-5

12. Alderman EL, Kip KE, Whitlow PL, et al. Native coronary disease progression exceeds failed revascularization as cause of angina after five years in the Bypass Angioplasty Revascularization Investigation (BARI). J Am Coll Cardiol. 2004;44(4):766-774. doi:10.1016/j.jacc.2004.05.041
13. 2014 ESC/EACTS Guidelines on myocardial revascularization: The Task Force on Myocardial Revascularization of the European Society of Cardiology (ESC) and the European Association for Cardio-Thoracic Surgery (EACTS)Developed with the special contribution of the European Association of Percutaneous Cardiovascular Interventions (EAPCI). Eur Heart J. 2014;35(37):2541-2619. doi:10.1093/eurheartj/ehu278

14. American Diabetes Association. 9. Cardiovascular Disease and Risk Management: Standards of Medical Care in Diabetes-2018. Diabetes Care. 2018;41(Suppl 1):S86-S104. doi:10.2337/dc18-S009

15. CholesterolTreatment Trialists'(CTT) Collaborators, Kearney PM, Blackwell L, et al. Efficacy of cholesterol-lowering therapy in 18,686 people with diabetes in 14 randomised trials of statins: a meta-analysis. Lancet Lond Engl. 2008;371(9607):117-125. doi:10.1016/50140-6736(08)60104-X

16. Cannon CP, Blazing MA, Giugliano RP, et al. Ezetimibe Added to Statin Therapy after Acute Coronary Syndromes. N Engl J Med. 2015;372(25):23872397. doi:10.1056/NEJMoa1410489

17. Sabatine MS, Leiter LA, Wiviott SD, et al. Cardiovascular safety and efficacy of the PCSK9 inhibitorevolocumabinpatients with and withoutdiabetes and the effect of evolocumab on glycaemia and risk ofnew-onset diabetes: a prespecified analysis of the FOURIER randomised controlled trial. Lancet Diabetes Endocrinol. 2017;5(12):941-950. doi:10.1016/52213-8587(17)30313-3

18. HPS3/TIMI55-REVEAL Collaborative Group, Bowman L, Hopewell JC, etal. Effects of Anacetrapib in Patients with Atherosclerotic Vascular Disease. N Engl J Med. 2017:377(13):1217-1227. doi:10.1056/NEJMoa1706444

19. NichollsSJ, Puri R, Anderson T, etal. Effect of Evolocumab on Progression of Coronary Disease in Statin-Treated Patients: The GLAGOV Randomized Clinical Trial. JAMA. 2016;316(22):2373-2384. doi:10.1001/jama.2016.16951

20. American Diabetes Association. 6. Glycemic Targets: Standards of Medical Care in Diabetes-2018. Diabetes Care. 2018;41(Suppl 1):S55-S64. doi:10.2337/dc18-S006

21. Skyler JS, Bergenstal R, Bonow RO, et al. Intensive glycemic control and the prevention of cardiovascular events: implications of the ACCORD, ADVANCE, and VA diabetes trials: a position statement of the American Diabetes Association and a scientific statement of the American College of Cardiology Foundation and the American Heart Association. Circulation. 2009;119(2):351-357. doi:10.1161/CIRCULATIONAHA.108.191305

22. Nathan DM, Cleary PA, Backlund J-YC, et al. Intensive diabetes treatment and cardiovascular disease in patients with type 1 diabetes. N Engl I Med. 2005;353(25):2643-2653. doi:10.1056/NEJMoa052187

23. Holman RR, Paul SK, Bethel MA, Matthews DR, Neil HAW. 10-year follow-up of intensive glucose control in type 2 diabetes. N Engl I Med. 2008;359(15):1577-1589. doi:10.1056/NEJMoa0806470 
24. LiU G, Li Y, Hu Y, et al. Influence of Lifestyle on Incident Cardiovascular Disease and Mortality in Patients With Diabetes Mellitus. J Am Coll Cardiol. 2018;71(25):2867-2876. doi:10.1016/j.jacc.2018.04.027

25. American Diabetes Association. 8. Pharmacologic Approaches to Glycemic Treatment: Standards of Medical Care in Diabetes-2018. Diabetes Care. 2018;41(Supp/ 1):S73-S85. doi:10.2337/dc18-S008

26. Everett $B M$, Donath MY, Pradhan AD, et al. Anti-Inflammatory Therapy With Canakinumab for the Prevention and Management of Diabetes. J Am Coll Cardiol. 2018;71(21):2392-2401. doi:10.1016/j.jacc.2018.03.002

27. Armstrong EJ, Rutledge JC, Rogers JH. Coronary Artery Revascularization in Patients with Diabetes. Circulation. 2013;128(15):1675-1685. doi:10.1161/CIRCULATIONAHA. 113.002114

28. UchidaT,PopmaJ,Stone GW, etal. The clinical impact of routine angiographic follow-up in randomized trials of drug-eluting stents: a critical assessment of "oculostenotic" reintervention in patients with intermediate lesions. JACC Cardiovasc Interv. 2010;3(4):403-411. doi:10.1016/.j.jin.2010.01.010

29. Navarese EP, Tandjung $K$, Claessen B, et al. Safety and efficacy outcomes of first and second generation durable polymer drug eluting stents and biodegradable polymer biolimus eluting stents in clinical practice: comprehensive network meta-analysis. BMJ. 2013;347:f6530. doi:10.1136/bmj.f6530

30. Kang S-H, Chae I-H, Park J-J, et al. Stent Thrombosis With Drug-Eluting Stents and Bioresorbable Scaffolds: Evidence From a Network Meta-Analysis of 147 Trials. JACC Cardiovasc Interv. 2016:9(12):1203-1212. doi:10.1016/j.jcin.2016.03.038

31. Steg $P G$, Harrington RA, Emanuelsson $\mathrm{H}$, et al. Stent thrombosis with ticagrelor versus clopidogrel in patients with acute coronary syndromes: an analysis from the prospective, randomized PLATO trial. Circulation. 2013;128(10):1055-1065. doi:10.1161/CIRCULATIONAHA.113.002589

32. Wiviott SD, Braunwald E, McCabe CH, et al. Intensive oral antiplatelet therapy for reduction of ischaemic events including stent thrombosis in patients with acute coronary syndromes treated with percutaneous coronary intervention and stenting in the TRITON-TIMI 38 trial: a subanalysis of a randomised trial. Lancet Lond Engl. 2008;371(9621):1353-1363. doi:10.1016/50140-6736(08)60422-5

33. Alsidawi S, Effat M, Rahman S, Abdallah M, Leesar M. The Role of Vascular Imaging in Guiding Routine Percutaneous Coronary Interventions: A Meta-Analysis of Bare Metal Stent and Drug-Eluting Stent Trials. Cardiovasc Ther. 2015;33(6):360-366. doi:10.1111/1755-5922.12160

34. RoyP, Steinberg DH, Sushinsky SJ, et al. The potential clinical utility of intravascular ultrasound quidance in patients undergoing percutaneous coronary intervention with drug-eluting stents. Eur Heart J. 2008;29(15):18511857. doi:10.1093/eurheartj/ehn249

35. LightellDJ, Woods TC. Relativeresistance to Mammalian targetofrapamycin inhibition in vascular smooth muscle cells of diabetic donors. Ochsner J. 2013;13(1):56-60

36. Centers forDisease Controland Prevention(CDC). Prevalenceofoverweight and obesity among adults with diagnosed diabetes--United States, 19881994 and 1999-2002. MMWR Morb Mortal Wkly Rep. 2004;53(45):10661068

37. Shan J, Nguyen TB, Totary-Jain H, Dansky H, Marx SO, Marks AR. Leptin-enhanced neointimal hyperplasia is reduced by mTOR and PI3Kinhibitors. Proc Natl Acad Sci. 2008;105(48):19006-19011. doi:10.1073/pnas.0809743105

38. Kim W-J, Lee S-W, Park S-W, et al. Randomized comparison of everolimus-eluting stent versus sirolimus-eluting stent implantation for de novo coronary artery disease in patients with diabetes mellitus (ESSENCE-DIABETES): results from the ESSENCE-DIABETES trial. Circulation. 2011;124(8):886-892. doi:10.1161/CIRCULATIONAHA.110.015453

39. Park KW, LeeJM, Kang S-H, et al. Everolimus-eluting Xiencev/Promus versus zotarolimus-eluting resolute stents in patients with diabetes mellitus. JACC Cardiovasc Interv. 2014;7(5):471-481. doi:10.1016/j.jcin.2013.12.201

40. Jensen LO, Thayssen P, Junker A, et al. Comparison of outcomes in patients with versus without diabetes mellitus after revascularization with everolimus- and sirolimus-eluting stents (from the SORT OUT IV trial). Am J Cardiol. 2012;110(11):1585-1591. doi:10.1016/j.amjcard.2012.07.022
41. GrubeE, ChevalierB, GuagliumiG, etal. TheSPIRITV diabeticstudy: arandomized clinical evaluation of the XIENCEV everolimus-elutingstentvs the TAXUS Libertépaclitaxel-eluting stent in diabetic patients with de novo coronary artery lesions. Am Heart J. 2012;163(5):867-875.e1. doi:10.1016/j.ahj.2012.02.006

42. BangaloreS, Bhagwat A, Pinto B, etal. Percutaneous Coronary Intervention in Patients With Insulin-Treated and Non-Insulin-Treated Diabetes Mellitus: Secondary Analysis of the TUXEDO Trial. JAMA Cardiol. 2016;1(3):266273. doi:10.1001/jamacardio.2016.0305

43. Silber S, Serruys PW, Leon MB, et al. Clinical outcome of patients with and without diabetes mellitus after percutaneous coronary intervention with the resolute zotarolimus-eluting stent: 2-year results from the prospectively pooled analysis of the international global RESOLUTE program. JACC Cardiovasc Interv. 2013;6(4):357-368. doi:10.1016/j.jcin.2012.11.006

44. Nakatsuma K, Shiomi $H$, Natsuaki $M$, et al. Second-generation versus first-generation drug-eluting stents in patients with and without diabetes mellitus: pooled analysis from the RESET and NEXT trials. CardiovasC Interv Ther. 2018;33(2):125-134. doi:10.1007/s12928-017-0458-9

45. Kufner S, ByrneRA,Mehilli, etal. Second-versusfirst-generation "Limus"-eluting stents in diabetic patients with coronary artery disease: a randomized comparison in setting of ISAR-TEST-4 trial. Catheter Cardiovasc Interv Off J Soc Card Angiogr Interv. 2013;82(6):E769-776. doi:10.1002/ccd.24741

46. Harada Y, Colleran R, Kufner S, et al. Five-year clinical outcomes in patients with diabetes mellitus treated with polymer-free sirolimus- and probucol-eluting stents versus second-generation zotarolimus-eluting stents: a subgroup analysis of a randomized controlled trial. Cardiovasc Diabetol. 2016;15(1):124. doi:10.1186/s12933-016-0429-y

47. Olesen KKW, Tilsted $H-H$, Jensen $L O$, et al. Long-term outcome of sirolimus-eluting and zotarolimus-eluting coronary stent implantation in patients with and without diabetes mellitus (a Danish organization for randomized trials on clinical outcome III substudy). Am I Cardiol. 2015;115(3):298-302. doi:10.1016/i.amjcard.2014.10.038

48. Maeng M, Baranauskas A, Christiansen EH, et al. A 10-month angiographic and 4-year clinical outcome of everolimus-eluting versus sirolimus-eluting coronary stents in patients with diabetes mellitus (the DiabeDES IV randomized angiography trial). Catheter Cardiovasc Interv OffJ SOC Card Angiogr Interv. 2015;86(7):1161-1167. doi:10.1002/ccd.25875

49. Vardi M, Burke DA, BangaloreS, et al. Long-term efficacy and safety of Zotarolimus-eluting stent in patients with diabetes mellitus: pooled 5-year results from the ENDEAVOR III and IV trials. Catheter Cardiovasc Interv Off J Soc Card Angiogr Interv. 2013;82(7):1031-1038. doi:10.1002/ccd.25045

50. Dangas GD, Serruys PW, Kereiakes DJ, et al. Meta-analysis of everolimus-eluting versus paclitaxel-eluting stents in coronary artery disease: final 3-year results of the SPIRIT clinical trials program (Clinical Evaluation of the Xience V Everolimus Eluting Coronary Stent System in the Treatment of Patients With De Novo Native Coronary Artery Lesions). JACC Cardiovasc Interv. 2013;6(9):914-922. doi:10.1016/j.jcin.2013.05.005

51. Gilham D, Wasiak S, Tsujikawa LM, et al. RVX-208, a BET-inhibitor for treating atherosclerotic cardiovascular disease, raises ApoA-I/HDL and represses pathways that contribute to cardiovascular disease. Atherosclerosis. 2016;247:48-57. doi:10.1016/j.atherosclerosis.2016.01.036

52. Ringvold HC, Khalil RA. Protein Kinase C as Regulator of Vascular Smooth Muscle Function and Potential Target in Vascular Disorders. Adv Pharmacol San Diego Calif. 2017;78:203-301. doi:10.1016/bs.apha.2016.06.002

53. Liu Z, Khalil RA. Evolving mechanisms of vascular smooth muscle contraction highlight key targets in vascular disease. Biochem Pharmacol. 2018;153:91-122. doi:10.1016/j.bcp.2018.02.012

54. Ali ZA, Brinton TJ, Hill JM, et al. Optical Coherence Tomography Characterization of Coronary Lithoplasty for Treatment of Calcified Lesions: First Description. JACC Cardiovasc Imaging. 2017;10(8):897-906. doi:10.1016/j. jcmg.2017.05.012

55. Byrne RA, Banai S, Colleran R, Colombo A. Challenges in Patients with Diabetes: Improving Clinical Outcomes After Percutaneous Coronary Intervention Through EVOlving Stent Technology. Interv Cardiol Lond Engl. 2018;13(1):40-44. doi:10.15420/icr.2017:27:1 